\title{
THE MEET OF LOCALLY CONNECTED GROUP TOPOLOGIES
}

\author{
DORENE J. FOX
}

Louisiana State University at Alexandria

8100 Hwy 71 South, Alexandria LA 71302

(Received November 6, 1992 and in revised form March 23, 1993)

ABSTRACT. The collection of all group topologies on a given group is a complete lattice partially ordered by inclusion. The purpose of this paper is to develop a convenient method of defining the meet of an arbitrary collection of group topologies and demonstrate that the meet of a collection of locally connected group topologies is also locally connected.

KEY WORDS AND PHRASES. Topological groups, quotient spaces, locally connected, translatable and semicontinuous topologies.

1992 AMS SUBJECT CLASSIFICATION CODES. 22A05, 54B15.

1. INTRODUCTION.

There has been considerable interest, by Graev [1], Comfort [2] and others, in finding group topologies on a given group. An approach is to start with a topology on a given group and derive an associated group topology. In [3] Roelcke and Dierolf developed a method for finding a neighborhood basis for the finest group topology contained in the intersection of an arbitrary collection of topologies on a group. In general, however, this basis does not consist of open sets. In [4] $\mathrm{Clark}$ and Schneider studied the meet in the lattice of group topologies and developed a convenient method for finding a basis of open sets for the meet of a countable collection of group topologies on an abelian group.

In this paper we will develop a convenient method for finding an open neighborhood basis for the finest group topology contained in any topology on a group. This method will enable us to find a neighborhood basis for the meet of an arbitrary collection of group topologies and demonstrate that the meet of an arbitrary collection of locally connected group topologies is also locally connected.

2. PRELIMINARY RESULTS.

Let $G$ be a group. Then it is well known that $\mathscr{F}$ is a fundamental system for $G$ iff $F$ is a collection of subsets of $G$ each containing the identity and satisfying the following properties:

1) If $U, V \in \mathscr{F}$ then there exists $W \in \mathscr{F}$ such that $W \subset U \cap V$.

2) If $U \in \mathscr{F}$ and $a \in U$ then there exists $V \in \mathcal{F}$ such that aV $\subset U$.

3) If $U \in \mathscr{F}$ and $x \in G$ then there exists $V \in \mathcal{F}$ such that $x V x^{-1} \subset U$.

4) If $U \in \mathscr{F}$ then there exists $V \in \mathscr{F}$ such that $V^{-1} \subset U$.

5) If $U \in \mathscr{F}$ then there exists $V \in \mathscr{F}$ such that $V V \subset U$.

If $\mathscr{F}$ is a fundamental system then there exists a unique topology $t$ on $G$ such that $t$ is a group topology having $F$ as a basis at the identity.

DEFINITION 1. Let $G$ be a group and $\mathcal{T}$ be a collection of subsets of $G$ each containing the identity of $G$. Then $\mathcal{T}$ is a translatable system for $G$ iff $\mathcal{T}$ satisfies properties 1,2 and 3 above and by [5] $\mathfrak{T}$ is a semifundamental system for $G$ iff $\mathfrak{J}$ satisfies properties 1 through 4 above.

In [5] Clay defined a semicontinuous topology and showed that a basis of open 
neighborhoods at the identity is a semifundamental system. She also showed that if $\varphi$ is a semifundamental system on a group $G$ then there is a unique topology $t$ on $G$ such that $t$ is a semicontinuous topology having $\varphi$ as a basis at the identity.

DEFINITION 2. A translatable group is a group $G$ endowed with a topology $t$ such that $\mathrm{Ua}$ and $\mathrm{aU}$ are elements of $t$ for all $U \in t$ and $a \in G$. If ( $\mathrm{G}, \mathrm{t})$ is $a$ translatable group then we say that $t$ is a translatable topology on $G$.

It is easy to see that if $t$ is a translatable topology then a basis of open neighborhoods at the identity is a translatable system and if $\mathcal{T}$ is a translatable system for a group $G$ then there is a unique topology $t$ on $G$ such that $t$ is a translatable topology having $\mathcal{J}$ as a basis at the identity.

DEFINITION 3. Let $G$ be a group and $t$ any topology on $G$. The Graev operator assigns to the pair $(G, t)$ the pair $(G, g(t))$ where $g(t)$ is the finest group topology on $G$ contained in $t$.

The collection of all group topologies on a given group is a complete lattice partially ordered by inclusion. The join of an arbitrary collection of group topologies $\left\{\mathrm{t}_{\alpha}\right\}_{\alpha \in \Delta}$ has $\left\{\mathrm{V}: \mathrm{V} \in \mathrm{t}_{\alpha}\right.$ for some $\left.\alpha \in \Delta\right\}$ as a subbasis and the meet is the join of $\left\{t: t<t_{\alpha}\right.$ for all $\alpha \in \Delta$ and $t$ is a group topology\}. Samuel pointed out in [6] that $\hat{n}_{\alpha} \mathrm{t}_{\alpha}$ may not be a group topology. Therefore $\hat{\alpha \in \Delta}_{\alpha}^{\mathrm{t}} \neq \hat{\alpha \in \Delta}_{\alpha} \mathrm{t}_{\alpha}$ (in general) but $g\left(\hat{\alpha \in \Delta}_{\alpha} \mathrm{t}_{\alpha}\right) \mathrm{t}_{\alpha}$ for all $\alpha \in \Delta$ and $g\left({ }_{\alpha \in \Delta} \mathrm{t}_{\alpha}\right)$ is a group topology. Therefore $g\left(\hat{\alpha \in \Delta}_{\alpha} \mathrm{t}\right)=\underset{\alpha \in \Delta}{\mathrm{t}} \alpha$.

If $G_{1}=G$ for all $i \in \mathbb{N}$ then we let $\underset{1=1}{\oplus \infty} G$ denote $\underset{1=1}{\oplus_{1}^{\infty} G}, \prod_{1=1}^{\infty} G$ denote $\prod_{1=1}^{\infty} G_{1}$ and define $m_{\infty}: \oplus_{1=1}^{\infty} G \longrightarrow G$ by $m_{\infty}\left(<a_{1}>\right)=a_{1} a_{2} a_{3} \cdots a_{n}$ where $a_{m}=$ e for all $m>n$. Let $T$ be a translatable topology on $\underset{1=1}{\oplus} G$, $t$ be a translatable topology on $G$, and $S$ be the topology inherited by $\underset{i=1}{\oplus \infty} \mathrm{G}$ as a subspace of $\prod_{i=1}^{\infty} G$ when $\prod_{i=1}^{\infty} G$ is endowed with the box topology with $t$ on each factor. Then $q_{\infty}(T)$ shall denote the quotient topology on $G$ induced by $m_{\infty}:(\underset{1=1}{\oplus} G, T) \longrightarrow G$ and $q_{\infty}[t]$ shall denote the quotient topology $q_{\infty}(S)$.

THEOREM 1. If $T$ is a translatable topology on $\oplus_{1=1}^{\infty} G$ then the quotient map $m_{\infty}:(\stackrel{\oplus=1}{\oplus} G, T) \longrightarrow\left(G, q_{\infty}(T)\right)$ is open.

PROOF: Let $V \in T$ and $\left\langle a_{1}\right\rangle \in m_{\infty}^{-1}\left(m_{\infty}(V)\right)$ then there exists $\left\langle b_{1}\right\rangle \in V$ such that $m_{\infty}\left(\left\langle b_{1}\right\rangle\right)=m_{\infty}\left(\left\langle a_{1}\right\rangle\right)$. Since $\left\langle a_{1}\right\rangle$ and $\left\langle b_{1}\right\rangle$ are in $\oplus_{1=1}^{\infty} G$ there exists $m$ such that $a_{n}=e$ and $b_{n}=e$ for all $n>m$ and $b_{1} b_{2} b_{3} \cdots b_{m}=a_{1} a_{2} a_{3} \cdots a_{m}$.

Let $h_{1}=b_{1}^{-1} a_{1}$ and $h_{1}=b_{1} b_{1+1} b_{1+2} \cdots b_{m m} a_{m-1}^{-1} a_{m-2}^{-1} a^{-1} \cdots a_{1}^{-1}$ for all $i=3,4,5, \cdots m$. Let $\mathrm{u}=\left\langle\mathrm{e}, \mathrm{h}_{1}^{-1}, \mathrm{~h}_{3}^{-1}, \mathrm{~h}_{4}^{-1}, \cdots, \mathrm{h}_{\mathrm{m}-1}^{-1}, \mathrm{~h}_{\mathrm{m}}^{-1}, \mathrm{e}, \mathrm{e}, \mathrm{e}, \cdots\right\rangle$ and $\mathrm{w}=\left\langle\mathrm{h}_{1}, \mathrm{~h}_{3}, \mathrm{~h}_{4}, \cdots, \mathrm{h}_{\mathrm{m}}, \mathrm{e}, \mathrm{e}, \mathrm{e}, \cdots\right\rangle$ then $u, w \in \underset{i=1}{\oplus} \mathrm{G}$ and $\left\langle\mathrm{a}_{1}\right\rangle=\mathrm{u}\left\langle\mathrm{b}_{1}\right\rangle \mathrm{w} \in \mathrm{uVw} \in \mathrm{T}$ since $\mathrm{T}$ is translatable.

Let $\left\langle\mathrm{x}_{1}\right\rangle \in \mathrm{uVw}$. Then there exists $\left\langle\mathrm{y}_{1}\right\rangle \in \mathrm{V}$ such that $\left\langle\mathrm{x}_{1}\right\rangle=\mathrm{u}\left\langle\mathrm{y}_{1}\right\rangle \mathrm{w}$ and there exists $n_{y}$ such that $y_{1}=e$ for all $i>n_{y}$. Let $k=\max \left\{m, n_{y}\right\}$ then:

$$
\begin{aligned}
\left.m_{\infty}\left(<x_{1}\right\rangle\right)=x_{1} x_{2} x_{3} \cdots x_{k} & =\left(y_{1} h_{1}\right)\left(h_{1}^{-1} y_{2} h_{3}\right)\left(h_{3}^{-1} y_{3} h_{4}\right) \cdots\left(h_{m-1}^{-1} y_{m-1} h_{m}\right)\left(h_{m}^{-1} y_{m}\right) y_{m+1} y_{m+1} \cdots y_{k} \\
& =y_{1} y_{2} y_{3} \cdots y_{k} \in m_{\infty}(V)
\end{aligned}
$$

COROLLARY 1. Let $n \in \mathbb{N}$ and define $m_{n}: \prod_{i=1}^{n} G \longrightarrow G$ by $m_{n}\left(a_{1}, a_{2}, a_{3}, \ldots a_{n}\right)=$ $a_{1} a_{2} a_{3} \cdots a_{n}$. Let $T$ be a translatable topology on $\prod_{i=1}^{n} G$ then the quotient map $m_{n}:\left(\prod_{1=1}^{n} G, T\right) \longrightarrow G$ is open. 
3. THE GRAEV OPERATOR.

Let $n \in\{0,1,2, \ldots\}, P_{n}$ be the set of all permutations on $\{n+1, n+2, n+3, \ldots .$. and $\sigma_{1}$ denote $\sigma^{-1}(i)$ for all $i \in \mathbb{N}$ and $\sigma \in P_{n}$. Suppose $s$ is a semicontinuous topology on $G$ and $\mathscr{S}$ is a semifundamental system for $\mathrm{s}$. Let $\left\langle\mathrm{A}_{i}\right\rangle$ be a sequence of elements of $\varphi$ and:

$P\left(\left\langle A_{1}\right\rangle, n\right)=\underset{\substack{\sigma \in \mathbb{P}_{n} \\ j \in \mathbb{N}}}{ }\left(A_{1} \times A_{2} \times A_{3} \times \cdots \times A_{n} \times A_{\sigma_{n+1}} \times A_{\sigma_{n+2}} \times \cdots \times A_{\sigma_{n+j}} \times e \times e \cdots\right)$

Then $\left\{P\left(\left\langle A_{1}\right\rangle, n\right): A_{1} \in \varphi\right.$ and $\left.n \in\{0,1,2, \ldots\}\right\}$ is a translatable system for a topology, $\mathrm{C}_{s}$, on $\stackrel{\oplus}{1=1}^{\infty} \mathrm{G}$. (Introduced by Bradd Clark, $\mathrm{C}_{s}$ is known as the cross topology due to the "shape" of the basic neighborhoods of the identity element.) Now if we let $C\left(\left\langle A_{1}\right\rangle\right)=\underset{\sigma \in P_{c}}{U}\left[\bigcup_{n \in \mathbb{N}}\left(A_{\sigma_{1}} A_{\sigma_{2}} A_{\sigma_{3}} \cdots A_{\sigma_{n}}\right)\right]$ then $C(s)=\left\{C\left(\left\langle A_{1}\right\rangle\right): A_{1} \in \varphi\right\}$ is a neighborhood basis of the identity for $q_{\infty}\left(C_{s}\right)$. This follows from the fact that the quotient map $m_{\infty}:\left(\stackrel{\oplus}{\stackrel{\infty}{1} G}, C_{s}\right) \longrightarrow G$ is open.

THEOREM 2. $q_{\infty}\left(C_{s}\right)=g(s)$.

PROOF: One can easily see that $C(s)$ satisfies properties 1,3 and 4 of a fundamental system. Therefore let us consider properties 2 and 5 . Let $U=C\left(\left\langle U_{1}\right\rangle\right)$ be an element of $C(s)$ and $a \in U$ then:

(2) There exists $n \in \mathbb{N}$ and $\delta \in P_{0}$ such that $a \in U_{\delta_{1}} U_{\delta_{2}} U_{\delta_{3}} \cdots U_{\delta_{n}}$. Therefore we can $f$ ind $a_{1} \in U_{\delta_{1}}$ for $i=1,2,3, \ldots, n$ such that $a=a_{1} a_{2} a_{3} \ldots a_{n}$ and we can find $v_{1} \in \varphi$ such that $v_{1} a_{1} \subset U_{\delta_{1}}$ for all $i=1,2,3, \ldots, n$. Let $b_{1}=a_{1} a_{1+1} a_{1+2} \cdots a_{n}$ then there exists $U_{1}^{\prime} \in \varphi$ and $W \in \varphi$ such that $b_{1} U_{1}^{\prime} \subset V_{1} b_{1}$ for all $i=1,2,3, \ldots, n$ and $W \subset \underset{1=1}{n} U_{1}^{\prime}$. Now $U_{\delta_{1}} U_{\delta_{2}} U_{\delta_{3}} \cdots U_{\delta_{n}} \supset \mathrm{WW}^{\mathrm{n}}$ and we can find, for all $\mathrm{i} \in \mathbb{N}, W_{1} \in \varphi$ such that $W_{1} \subset U_{\delta_{n+1}} \cap W^{n}$. Therefore $a\left[C\left(\left\langle W_{1}\right\rangle\right)\right] \subset U$.

(5) There exists $v_{1} \in \varphi$ for $i=1,3,5, \ldots$ such that $v_{1} \subset U_{1} \cap U_{1+1}$. Let:

$$
\left\langle A_{1}\right\rangle=\left\langle U_{1}, U_{3}, U_{5}, \ldots\right\rangle, \quad\left\langle B_{1}\right\rangle=\left\langle U_{2}, U_{4}, U_{6}, \ldots\right\rangle \text { and } \mathrm{V}=\mathrm{C}\left(\left\langle\mathrm{V}_{1}\right\rangle\right) \text {. }
$$

Then $\mathrm{V}^{2} \subset\left[C\left(<\mathrm{A}_{1}>\right)\right]\left[\mathrm{C}\left(<\mathrm{B}_{1}>\right)\right] \subset \mathrm{U}$.

Therefore $q_{\infty}\left(C_{s}\right)$ is a group topology and since $s$ is translatable $q_{\infty}\left(C_{s}\right) C s$. Let $U \in g(s)$ such that $e \in U$ and suppose $\varphi$ is the set of all open neighborhoods of e. Then there exists $V_{1} \in g(s)$ such that $e \in V_{1}$ and $\left(V_{1}\right)^{3} \subset U$. We can also find $V_{1} \in g(s)$ such that $e \in V_{1}$ and $\left(V_{1}\right)^{3} \subset V_{1-1}$ for all $i=2,3,4, \ldots$

Let $\sigma \in P_{0}$ and $k_{m, n}=\min \left\{\sigma_{m}, \sigma_{m+1}, \sigma_{m+2}, \ldots, \sigma_{n}\right\}\left(\sigma_{1} \operatorname{denotes} \sigma^{-1}(i)\right.$ for all $i \in \mathbb{N})$. Then $v_{\sigma_{1}} v_{\sigma_{2}} \subset\left(V_{k_{1,2}}\right)^{2} \subset\left(V_{k_{1,2}}\right)^{3} \subset U, V_{\sigma_{1}} V_{\sigma_{2}} v_{\sigma_{3}} \subset\left(V_{k_{1,3}}\right)^{3} \subset U$ and $\mathrm{V}_{\sigma_{1}} \mathrm{~V}_{\sigma_{2}} \mathrm{~V}_{\sigma_{3}} \mathrm{~V}_{\sigma_{4}} \subset\left(\mathrm{V}_{\mathrm{k}_{1,2}}\right)^{2}\left(\mathrm{~V}_{\mathrm{k}_{3,4}}\right)^{2}<\left(\mathrm{V}_{\mathrm{k}_{1,4}}\right)^{3}<\mathrm{U}$

Suppose then that $V_{\sigma_{1}} V_{\sigma_{2}} V_{\sigma_{3}} \cdots V_{\sigma_{1}} \subset\left(V_{k_{1,1}}\right)^{3} \subset U$ for all $i \leq n-1$ and $\sigma \in P_{0}$. Let $\sigma_{j}=k_{1, n}$ then: 


$$
\begin{aligned}
v_{\sigma_{1}} v_{\sigma_{2}} v_{\sigma_{3}} \cdots v_{\sigma_{n}} & =v_{\sigma_{1}} v_{\sigma_{2}} v_{\sigma_{3}} \cdots v_{\sigma_{j-1}} v_{\sigma_{j}} v_{\sigma_{j+1}} \cdots v_{\sigma_{n}} \\
& \subset\left(v_{k_{1, j-1}}\right)^{3} v_{k_{1, n}}\left(v_{k_{j+1, n}}\right)^{3} \subset\left(v_{k_{1, n}}\right)^{3} \subset U .
\end{aligned}
$$

Therefore $C\left(\left\langle V_{i}>\right) \subset U\right.$ and $g(s) \subset q_{\infty}\left(C_{s}\right) \subset s$. Therefore $g(s)=q_{\infty}\left(C_{s}\right)$ since $q_{\infty}\left(C_{s}\right)$ is a group topology .

If $G$ is abelian then $G(s)=\left\{\bigcup_{n \in \mathbb{N}}\left(V_{1} V_{2} V_{3} \ldots V_{n}\right) \mid V_{1} \in \varphi\right\}$ and by a proof similar to the one above we can prove the following corollary.

COROLLARY 2 If $G$ is abelian then $g(s)=q_{\infty}[s]$.

Suppose then that we have an arbitrary collection of group topologies $\left\{t_{\alpha}\right\}_{\alpha \in \Delta}$ on a given group. Since $\hat{n}_{\alpha} \mathrm{t}_{\alpha}$ is semicontinuous we have that $C\left(\hat{n}_{\alpha} \mathrm{t}_{\alpha}\right)$ is a fundamental system for $\hat{\alpha \in \Delta} \mathrm{t}_{\alpha}$.

In [7] Clark and Schneider defined $S(t)$ to be the finest semicontinuous topology on $G$ contained in $t$ and gave a concrete description of $S(t)$. Since $g(t)$ is semicontinuous $g(S(t))=g(t)$. Therefore we can also use Theorem 2 to prove the following corollary.

COROLLARY 3. If $t$ is any topology on a group $G$ and $C_{s(t)}$ is Clark's Cross topology with respect to $S(t)$ then $q_{\infty}\left(C_{S(t)}\right)=g(t)$.

4. LOCAL CONNECTEDNESS.

Suppose $A$ and $B$ are connected subsets of a translatable group $G$ then $a B$ is connected for all $a \in A$. Let $x \in B$ then $A x \subset A B$ and $A x$ is connected. Therefore $A B$ $=A x \cup A B=A x \cup\left[\bigcup_{a \in A}(a B)\right]$ is connected since $a x \in A x \cap a B$ for all $a \in A$. Therefore we have the following theorem.

THEOREM 3. Suppose $t$ is a locally connected and translatable topology on $G$ then $q_{\infty}[t]$ is also locally connected.

In a similar fashion we can prove the following corollary.

COROLLARY 4. If $t$ is a locally connected and translatable topology on $G$ and $C_{t}$ is Clark's Cross topology with respect to $t$ then $q_{\infty}\left(C_{t}\right)$ is also locally connected.

Suppose then that $\left\{t_{\alpha}\right\}_{\alpha \in \Delta}$ is a collection of locally connected group topologies

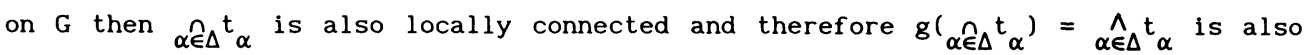
locally connected.

ACKNOWLEDGEMENTS. Many of these results were developed in the author's Dissertation, titled "Translatable Groups and the Graev Operator" under the direction of Bradd Clark, University of Southwestern Louisiana, 1991.

\section{REFERENCES}

1. GRAEV, M. Free Topological Groups, Izv. Akad Nauk SSSR Ser. Mat. 12 (1948), $279-324$.

2. COMFORT, W.W. and ROSS, Kenneth A. Topologies Induced by Groups of Characters, Fund. Math., Vol. 55 (1964), $283-291$.

3. ROELCKE, W. and DIEROLF, S. Uniform Structures on Topological Groups and Their Quotients, McGraw Hill, 1981.

4. CLARK, B. and SCHNEIDER, V. The Meet Operator in the Lattice of Group Topologies, Canad. Math. Bull. Vol. 29 (1986), $478-481$.

5. CLAY, E. Semicontinuous Groups, Dissertation, University of Southwestern Louisiana, 1990.

6. SAMUEL, P. Ultrafilters and Compactifications of Uniform Spaces, Trans. AMS 64 (1948), $110-132$.

7. CLARK, B. and SCHNEIDER, V. A Comparison of Topologies on Free Groups, To Appear. 


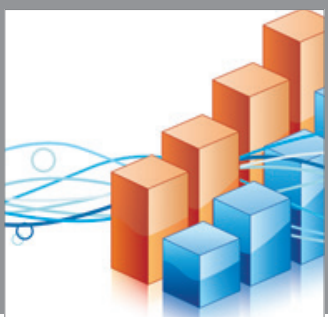

Advances in

Operations Research

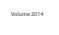

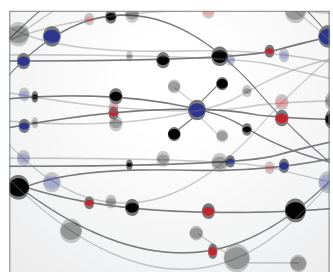

\section{The Scientific} World Journal
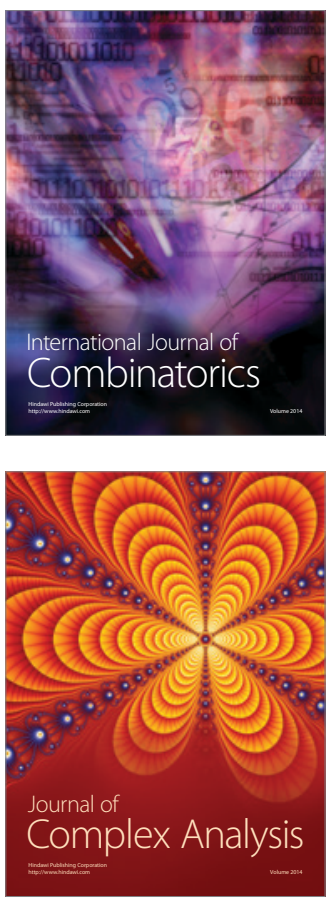

International Journal of

Mathematics and

Mathematical

Sciences
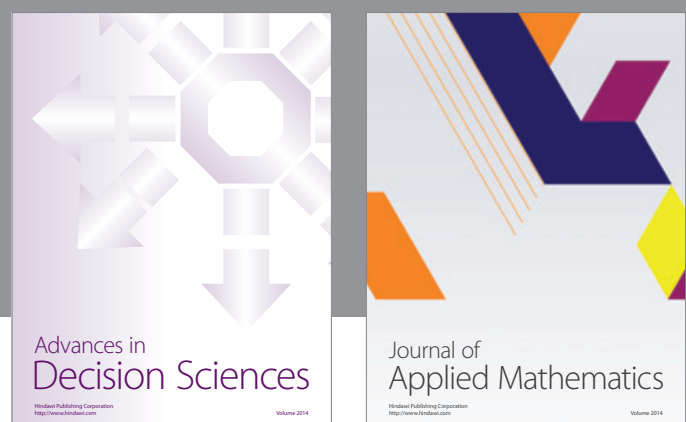

Journal of

Applied Mathematics
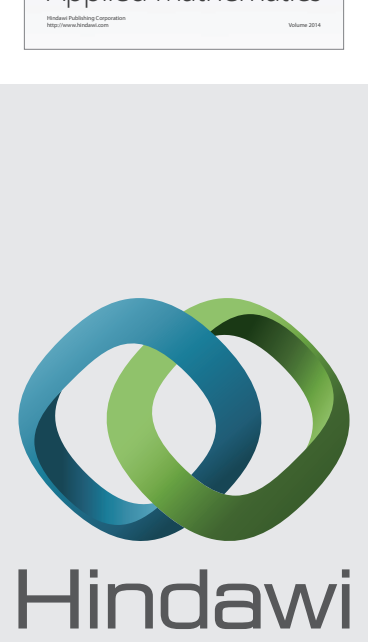

Submit your manuscripts at http://www.hindawi.com
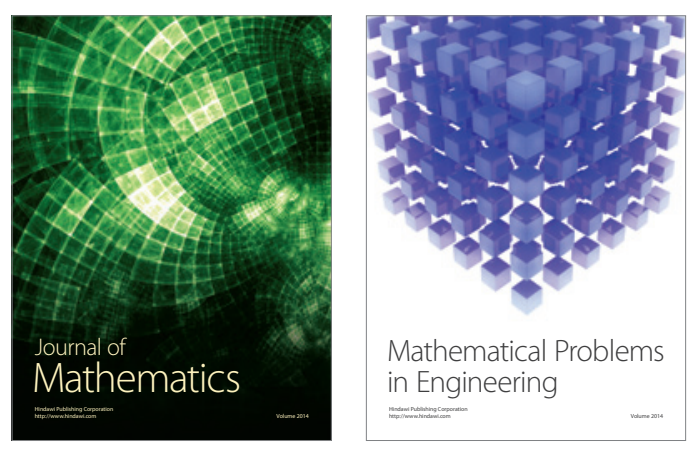

Mathematical Problems in Engineering
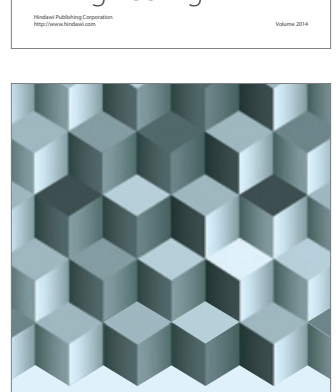

Journal of

Function Spaces
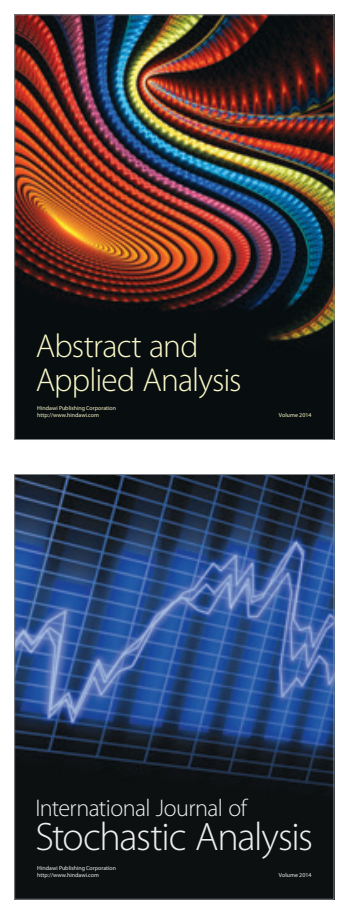

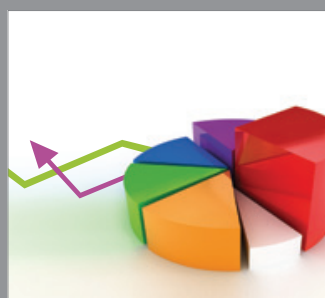

ournal of

Probability and Statistics

Promensencen
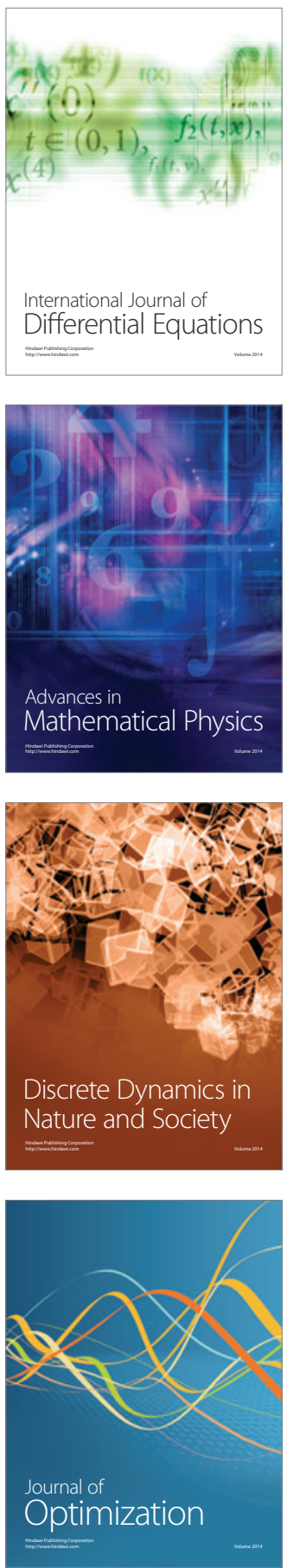